\title{
Myoblast-seeded biodegradable scaffolds to prevent post- myocardial infarction evolution toward heart failure
}

\author{
Matthias Siepe, MD, a,b , Marie-Noëlle Giraud, PhD, ${ }^{\mathrm{b}}$ Mladen Pavlovic, MD, ${ }^{\mathrm{c}}$ Concetina Receputo, \\ Friedhelm Beyersdorf, MD, PhD, ${ }^{a}$ Philippe Menasché, MD, PhD, ${ }^{d}$ Thierry Carrel, MD, ${ }^{b}$ and Hendrik T. Tevaearai, MD
}

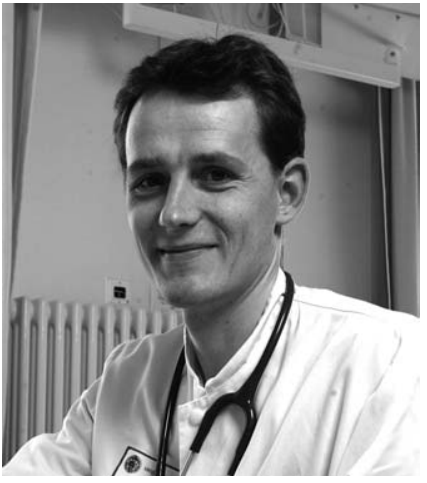

Dr Siepe
From the Clinic for Cardiovascular Surgery, University Hospital, ${ }^{\text {a }}$ Freiburg, Germany; the Clinics for Cardiovascular Surgery $^{\mathrm{b}}$ and Pediatric Cardiology, ${ }^{\mathrm{c}}$ University Hospital, Bern, Switzerland; and the Department of Cardiovascular Surgery, Hôpital Européen Georges Pompidou, ${ }^{d}$ Paris, France.

This work was supported by a grant from the Swiss National Foundation (\#3200065044). Dr Matthias Siepe was supported by the German Research Foundation, DFG, Kennedyallee 40, 53175 Bonn, Germany.

Received for publication Nov 3, 2005; revisions received Jan 7, 2006; accepted for publication Jan 18, 2006.

Address for reprints: Matthias Siepe, MD, Clinic for Cardiovascular Surgery, University Hospital Freiburg, Hugstetterstrasse 55, 79106 Freiburg, Germany (E-mail: matthias.siepe@web.de).

J Thorac Cardiovasc Surg 2006;132:124-31

$0022-5223 / \$ 32.00$

Copyright (๑) 2006 by The American Association for Thoracic Surgery

doi:10.1016/j.jtcvs.2006.01.052
Objective(s): Even though the mechanism is not clearly understood, direct intramyocardial cell transplantation has demonstrated potential to treat patients with severe heart failure. We previously reported on the bioengineering of myoblast-based constructs. We investigate here the functional outcome of infarcted hearts treated by implantation of myoblast-seeded scaffolds.

Methods: Adult Lewis rats with echocardiography-confirmed postinfarction reduced ejection fraction $(48.3 \% \pm 1.1 \%)$ were randomized to (1) implantation of myoblastseeded polyurethane patches at the site of infarction (PU-MyoB, $\mathrm{n}=11$ ), (2) implantation of nonseeded polyurethane patches (PU, $\mathrm{n}=11$ ), (3) sham operation (Sham, $\mathrm{n}=12$ ), and (4) direct intramyocardial myoblast injection (MyoB, $\mathrm{n}=11$ ). Four weeks later, the functional assessment by echocardiography was repeated, and we additionally performed left ventricular catheterization plus histologic studies.

Results: The ejection fraction significantly decreased in the PU $(39.1 \% \pm 2.3 \% ; P$ $=.02)$ and Sham $(39.9 \% \pm 3.5 \% ; P=.04)$ groups, whereas it remained stable in the PU-MyoB $(48.4 \% \pm 3.1 \%)$ and $\mathrm{MyoB}(47.9 \% \pm 3.0 \%)$ groups during the observation time. Similarly, left ventricular contractility was significantly higher in groups PU-MyoB $(4960 \pm 266 \mathrm{~mm} \mathrm{Hg} / \mathrm{s})$ and MyoB $(4748 \pm 304 \mathrm{~mm} \mathrm{Hg} / \mathrm{s})$ than in groups PU $(3909 \pm 248 \mathrm{~mm} \mathrm{Hg} / \mathrm{s}, P=.01)$ and Sham $(4028 \pm 199 \mathrm{~mm} \mathrm{Hg} / \mathrm{s}$, $P=.01)$. Immunohistology identified a high density of myoblasts within the seeded scaffolds without any migration toward the host cardiac tissue and no evidence of cardiac cell differentiation.

Conclusions: Myoblast-seeded polyurethane scaffolds prevent post-myocardial infarction progression toward heart failure as efficiently as direct intramyocardial injection. The immunohistologic analysis suggests that an indirect mechanism, potentially a paracrine effect, may be assumed.

\footnotetext{
A nimal studies have recently shown that intramyocardial injection of skeletal myoblasts can prevent the progressive hemodynamic alterations after a myocardial infarction (MI). ${ }^{1-3}$ These results, as well as the preliminary data from clinical phase I studies, ${ }^{4-6}$ are encouraging in a field where no treatment except organ transplantation has clearly demonstrated its efficacy. However, the myocardial injection process is associated with a dramatically high rate of immediate cell death that may potentially limit the benefits of this technique. ${ }^{7-9}$ One possible approach for overcoming this hurdle could be to use biodegradable scaffolds seeded with the candidate cell. $\mathrm{We}^{10}$ recently reported on the process of optimally seeding myoblasts on Artelon polyurethane (PU) scaffolds (Artimplant AB, Västra Frölunda, Sweden). In the current work, we have evaluated the possible functional benefit of these bioengineered constructs in a rat model of ischemic heart failure and compared this new approach with direct intramyocardial injection.
} 


\section{Abbreviations and Acronyms \\ $\mathrm{dP} / \mathrm{dt}_{\max }=$ left ventricular contractility \\ $\mathrm{dP} / \mathrm{dt}_{\min }=$ left ventricular relaxation \\ $\mathrm{EF} \quad=$ ejection fraction \\ LVEDP = left ventricular end-diastolic pressure \\ LVESP = left ventricular end-systolic pressure \\ LVEDV = left ventricular end-diastolic volume \\ LVESV = left ventricular end-systolic volume \\ MI = myocardial infarction \\ $\mathrm{PU}=$ polyurethane \\ PU-MyoB $=$ myoblast-seeded polyurethane patches at the site of infarction \\ $\mathrm{PU}=$ nonseeded polyurethane patches \\ MyoB = direct intramyocardial myoblast injection}

\section{Methods}

\section{Myoblast Culture}

Skeletal myoblasts were isolated and expanded from male newborn Lewis rats as described previously. ${ }^{11}$ In brief, limb muscles were minced and enzymatically dissociated with $1.5 \mathrm{mg} / \mathrm{mL}$ collagenase IA (Sigma Chemical Company, St Louis, Mo) and 0.25\% trypsine-ethylenediaminetetraacetic acid (Gibco, Auckland, New Zealand). After passage through 100- and 40- $\mu \mathrm{m}$ filters (Falcon; BD, Franklin Lakes, NJ), cells were expanded in culture medium consisting of 57\% minimum Eagle medium (Gibco), 27\% M199 (Gibco), 15\% fetal bovine serum (HyClone, Logan, Utah), 1\% P/S (Gibco), and $5 \mathrm{ng} / \mathrm{mL}$ beta fibroblast growth factor (Sigma). We harvested the cells by trypsinization after 48 hours, counted them, and froze them in culture medium containing $10 \%$ dimethyl sulfoxide (Sigma). An average of $14.4 \pm 2.2 \times 10^{6}$ cells were obtained per newborn Lewis rat. The proportion of skeletal myoblasts was estimated by anti-desmin (Monoclonal Anti Desmin, Clone DE-U-10; Sigma) immunolabeling as described previously. ${ }^{12}$ Culture purity reached $33.2 \% \pm 1.9 \%$ of desmin-positive myoblasts. Cells were later thawed, diluted, centrifuged, and counted before being seeded on PU scaffolds.

\section{Scaffold Preparation}

The manufacturing of Artelon PU scaffolds was described previously. ${ }^{13}$ In brief, Artelon is a long-term degradable biomaterial, which degrades by hydrolysis over a period of approximately 5 years. In this study, the biomaterial was processed into a highly porous scaffold $(90 \% \pm 3 \%$ volume porosity) with interconnected pores.

We prepared the scaffolds in groups of three: one seeded scaffold for implantation, one seeded scaffold for cell count and viability validation, and one unseeded scaffold. These unseeded scaffolds were incubated for 14 days in culture medium to obtain similar physical properties as compared with the implanted material.

For cell seeding, $7.5 \times 7.5 \times 2.0$-mm scaffolds were coated with $10 \mu \mathrm{g} / \mathrm{mL}$ laminin (Invitrogen, Carlsbad, Calif) before $4.9 \pm$ $1.2 \times 10^{6}$ cells were pipetted $(30-\mu \mathrm{L}$ suspension) over their surface. After a 30-minute attachment time, the seeded scaffolds were placed on silicone-coated dishes (Sylgard 184; Truller, Fu- lenbach, Switzerland) in 2-mL culture medium and incubated at $37^{\circ} \mathrm{C}$ for 14 days with medium changes every 60 hours. Our current seeding protocol was adopted after a previous systematic analysis of several seeding conditions was performed. ${ }^{10}$ In this preliminary study, spectrometic MTT viability test (MTT thiazolyl blue; Sigma), CyQuant Cell Proliferation Assay Kit (Molecular Probes, Eugene, Ore), and immunohistology were used to assess cell viability and cell number.

\section{Animal Model}

The animals received human care in compliance with the "Principles of Laboratory Animal Care" formulated by the National Society for Medical Research and the "Guide for the Care and Use of Laboratory Animals" prepared by the Institute of Laboratory Animal Resources and published by the National Institutes of Health (NIH Publication No. 86-23, revised 1985). All procedures were performed in accordance with the Swiss Animal Protection Law after obtaining the permission of the State Veterinary Office, Bern, Switzerland.

For the operative procedures, female Lewis rats (average weight: $203 \pm 1 \mathrm{~g}$ ) were anesthetized with isoflurane $(5 \%$ of oxygen for induction and $2.5 \%$ of oxygen for maintenance) and additive buprenorphine $(10 \mu \mathrm{g} / \mathrm{kg})$, placed on a warming pad $\left(37^{\circ} \mathrm{C}\right)$, and tracheally ventilated at $80 \mathrm{cycles} / \mathrm{min}$ (14-gauge intravenous cannula, Abbocath; Abbott, Sligo, Republic of Ireland; Small Animal Ventilator 683; Harvard Apparatus Inc, Holliston, Mass).

On day 0 , an MI was induced through a left lateral thoracotomy by ligation of the left anterior descending coronary artery with a 7-0 polypropylene suture (Ethicon, Inc, Somerville, NJ). On day 14, a baseline echocardiographic analysis was performed before the animals were randomized to receive either a sham operation (group Sham $=$ sternotomy and adhesiolysis only, $\mathrm{n}=11$ ), the implantation of a myoblast-seeded (PU-MyoB, $n=11$ ) or nonseeded scaffold (PU, $\mathrm{n}=12$ ), or the direct intramyocardial injection of a myoblast suspension (MyoB, $\mathrm{n}=11$ ), or medium only (Medium, $\mathrm{n}=10$ ). All interventions were performed through a median sternotomy. The scaffolds were attached to the outer surface of the myocardial scar with 4 single-knot sutures connecting the PU scaffold edges with the infarction area edges. In the MyoB group of animals, $4.8 \pm 0.9 \times 10^{6}$ cells in $150-\mu \mathrm{L}$ medium (containing 15\% fetal bovine serum) were distributed within the scar via 4 to 6 intramyocardial injection sites. Four weeks after the intervention (day 42), the rats were anesthetized for echocardiography and left ventricular pressure measurements before the hearts were explanted for histologic analysis.

We additionally included a supporting group of 7 normal age-matched rats without MI or other intervention to serve as references for the echocardiographic values.

\section{Functional Assessment by Echocardiography}

The echocardiographic analyses were performed in a blinded manner with a 9 to $11-\mathrm{MHz}$ linear array transducer system (Acuson Sequoia; Siemens Inc, Malvern, Pa). Animals were anesthetized with $2.5 \%$ isoflurane by mask and placed on the left lateral position. The ejection fraction (EF) was calculated as described previously. ${ }^{12}$ In brief, in a 2-dimensional long-axis view, the left ventricular end-diastolic (LVEDV) and end-systolic volumes 
TABLE 1. Animal statistics

\begin{tabular}{lccccrrr}
\hline & No MI & Sham & PU & PU-MyoB & MyoB & Medium & Total \\
\hline Death post intervention & & 2 & - & - & - & - \\
Death at pressure catheter & & - & - & - & - & - & 2 \\
Exclusion & 7 & 12 & 11 & 11 & - & - \\
Included number of & & & 11 & 1
\end{tabular}

animals

No MI, normal rats without myocardial infarction; Sham, sham operation; $P U$, implantation of nonseeded polyurethane patch; $P U$-MyoB, myoblast-seeded polyurethane patch; Medium, contrast medium only; death post intervention, animals died 2 and 5 days after intervention; Death at pressure catheter, the carotid artery ruptured, the catheter did not pass the aortic valve, or the animals died of arrhythmia; Exclusion, the rat had dehiscent and deep infection of the sternotomy wound with intrathoracic involvement.

(LVESV) were calculated by the single-plane area (A) - length (L) method: Volume $=8 \times A^{2} /(3 \times \pi \times L)$. The EF was then calculated as: $E F=(L V E D V-L V E S V) / L V E D V$. Heart rate was measured in a short-axis view.

\section{Postinterventional Catheterization}

The analysis was also performed in a blinded manner. The tip of a 1.4F pressure catheter (Millar Mikro-Tip; Millar Instruments, Inc, Houston, Tex) was placed via the right carotid artery into the left ventricle before the animals were positioned in the left lateral position. ${ }^{14}$ After a stabilization period of 5 minutes, the pressure curves of 10 to 15 subsequent arbitrarily chosen beats were recorded and used to calculate the left ventricular contractility (dP/ $\left.\mathrm{dt}_{\text {max }}\right)$, relaxation $\left(\mathrm{dP} / \mathrm{dt}_{\text {min }}\right)$, end-systolic pressure (LVESP), enddiastolic pressure (LVEDP), and heart rate.

\section{Histology}

The hearts were cut in 3-mm-thick slices orthogonal to the long axis of the heart axis before being frozen in O.C.T. compound (Tissue Tek, Sakura Finetek, Zoeterwoude, Netherlands) and stored at $-80^{\circ} \mathrm{C}$. We then prepared 5 - $\mu$ m-thick cryosections on slides fixed with paraformaldehyde and stained with hematoxylin and eosin or an immunostain. For the latter, the slides were blocked with $3 \%$ bovine serum albumin (Sigma), then incubated 80 minutes with either of the following primary antibodies: antidesmin (ready to use, Mouse Anti-Human Desmin MCA849HT; Serotec, Oxford, United Kingdom), anti- $\alpha$-actinin (sarcomeric) (1:800, Clone EA-53; Sigma), anti-skeletal myosin (fast) (1:400, clone MY-32; Sigma), and anti-skeletal myosin (slow) (MAB1628, 1:400; Chemicon International, Temecula, Calif). Thereafter, the slides were incubated for 30 minutes with a horseradishperoxidase-linked secondary antibody (1:50 F(ab')2 Rabbit Anti Mouse immunoglobulin G [AFF.PUR]:HRP, STAR 13B; Serotec). Coloration steps with AEC (A6926; Sigma) application and hematoxylin were then performed.

\section{Statistics}

Data were analyzed by a computerized statistical program (SigmaStat; SPSS Inc, Chicago, Ill). The results are expressed as mean \pm SE. One-way analysis of variance was used to compare treatment groups. If significant differences were detected, pair-wise multiple comparison procedures were then performed (HolmSidak method). A paired $t$ test was used to test the differences between preinterventional and postinterventional parameters. A $\chi^{2}$ test was used to analyze the mortality rate.

\section{Results \\ Preimplantation Seeded Scaffold Assessment}

Six hours after cell seeding, the CyQuant proliferation assay showed that $34.9 \% \pm 5.0 \%$ of delivered cells $\left(5 \times 10^{6}\right)$ were attached to the PU scaffold. After an incubation time of 14 days, cell count increased and peaked to $80.9 \% \pm$ $5.2 \%$ of the initially delivered cells $(P<.001$ vs 6 hours after seeding). Similarly, viability after 14 days' incubation increased by $29.8 \% \pm 2.5 \%$ as compared with 6 hours after seeding as assessed by MTT test $(P<.001)$. Purity of myoblasts in culture was unaffected during this incubation time.

\section{Mortality and Morbidity}

Of a total of 113 surgically treated animals, 62 could be included in this study $(54.9 \%)$. Twenty-eight rats $(24.7 \%)$ died immediately after MI of ventricular arrhythmia; 2 rats $(1.7 \%)$ died between the MI and the first echocardiographic study. We further excluded 15 animals (13.3\%) on the basis of an echocardiographic EF smaller than $30 \%$ (3 rats) or greater than $60 \%$ (12 rats). Three animals $(2.6 \%)$ died of bleeding during the intervention procedure. Two animals died on days 2 and 5 after the intervention in severe heart failure. Five animals that were included in the study died during the postinterventional catheterization (Table 1). Of these 5 animals, 4 died of fatal arrhythmia during catheter placement and 1 died of bleeding. Importantly, none of those last 7 animals dying after the intervention belonged to either the MyoB or the PU-MyoB groups, making the mortality rate of non-myoblast treated animals $20.0 \%(7 / 35)$ as compared with $0 \%(P<.05)$ in the myoblast-treated groups of animals. Finally, 1 PU-MyoB rat was excluded from the study owing to an extended intrathoracic wound infection.

\section{Echocardiographic Data}

In normal rats without MI (group no MI), EF was significantly higher and LVEDV and LVESV were significantly 
TABLE 2. Preinterventional hemodynamic data (echocardiography)

\begin{tabular}{lcccccc}
\hline & No MI $(\mathbf{n}=\mathbf{7})$ & Sham $(\mathbf{n}=\mathbf{1 2})$ & PU $(\mathbf{n}=\mathbf{1 1})$ & PU-MyoB $(\mathbf{n}=\mathbf{1 1})$ & MyoB $(\mathbf{n}=\mathbf{1 1})$ & Medium $(\mathbf{n}=\mathbf{1 0})$ \\
\hline EF & $72.9 \pm 3.5^{*}$ & $48.2 \pm 2.7$ & $48.3 \pm 2.1$ & $47.9 \pm 2.1$ & $47.4 \pm 3.0$ & $50.1 \pm 2.1$ \\
LVEDV & $0.294 \pm 0.032^{*}$ & $0.441 \pm 0.042$ & $0.400 \pm 0.035$ & $0.416 \pm 0.024$ & $0.437 \pm 0.044$ & $0.372 \pm 0.0212$ \\
LVESV & $0.092 \pm 0.010^{*}$ & $0.233 \pm 0.028$ & $0.210 \pm 0.021$ & $0.222 \pm 0.022$ & $0.251 \pm 0.035$ & $0.185 \pm 0.011$ \\
HR & $386 \pm 11$ & $387 \pm 15$ & $384 \pm 12$ & $360 \pm 8$ & $369 \pm 14$ & $370 \pm 8$ \\
BW & $214 \pm 4$ & $214 \pm 3$ & $215 \pm 3$ & $220 \pm 2$ & $217 \pm 3$ & $216 \pm 2$ \\
\hline
\end{tabular}

No MI, Normal rats without myocardial infarction; Sham, sham operation; $P U$, implantation of nonseeded polyurethane patch; $P U-M y o B$, myoblast-seeded polyurethane patch; Medium, contrast medium only; $E F$, ejection fraction $(\%)$; $L V E D V$, left ventricular end-diastolic volume $(\mathrm{mL}) ; L V E S V$, left ventricular end-systolic volume $(\mathrm{mL}) ; H R$, heart rate $(1 / \mathrm{min}) ; B W$, body weight $(\mathrm{g})$. Values are mean $\pm \mathrm{SE}$. $* \mathrm{P}<.001$ versus all groups before and after intervention.

lower than in all groups of animals with MI and consecutive intervention $(P<.001)$. The heart rate and body weight did not differ between rats with no MI and the randomized rats.

The preinterventional echocardiographic evaluation confirmed the absence of differences between the randomized groups regarding ventricular function $(\mathrm{EF})$, ventricular sizes (LVESV, LVEDV), or heart rate (Table 2).

At the postinterventional echocardiograpic assessment (Table 3), the ventricular function (EF) in the control groups Sham, PU, and Medium decreased significantly as compared with the preinterventional values $(P=.021$ for Sham, $P=.003$ for PU, $P=.013$ for Medium). As shown in Figure 1, the postinterventional EFs of the PU-MyoB and MyoB animals were significantly higher than those of the Sham, PU, and Medium groups (PUMyoB vs Sham, $P=.042$; PU-MyoB vs PU, $P=.034$; MyoB vs Medium, $P=.017$ ). A slight increase in LVEDV in groups Sham, PU, PU-MyoB, and Medium did not reach statistical significance as compared with preinterventional values (Figure 2). Conversely, end point LVESV was significantly higher in the PU $(P=$ $.021)$ and Medium $(P=.018)$ groups compared with preinterventional values, but without differences between the groups (Figure 3). The heart rate at the time of echocardiographic assessment was similar in all groups, and the body weight was significantly higher than preinterventional values in all groups.

\section{Catheterization}

The left ventricular contractility $\left(\mathrm{dP} / \mathrm{dt}_{\max }\right)$ and relaxation $(\mathrm{dP} /$ $\mathrm{dt}_{\min }$ ) were significantly better in the PU-MyoB group of animals than in sham-operated animals $(P=.010$ for contractility and $P=.049$ for relaxation) and PU animals $(P=.006$ and $P=.011$, respectively). Similarly, the MyoB group of animals had higher contractility and relaxation values as compared with the PU group $(P=.026$ for contractility and $P=$ .015 for relaxation). The values for the control group Medium did not significantly differ from those seen in the myoblast treatment groups or in the other control groups (Figure 4).

\section{Histology}

Before transplantation, cell viability $(>95 \%)$ and antidesmin positivity rate $(33.2 \pm 1.9)$ were not affected by the freezing/thawing process.

TABLE 3. Postinterventional hemodynamic data (echocardiography and left ventricular catheterization)

\begin{tabular}{|c|c|c|c|c|c|}
\hline & Sham $(n=12)$ & $\mathrm{PU}(\mathrm{n}=11)$ & PU-MyoB $(n=11)$ & MyoB $(n=11)$ & Medium $(n=10)$ \\
\hline EF & $40.1 \pm 2.8^{*}$ & $39.6 \pm 1.8^{*}$ & $48.4 \pm 3.1 \dagger \ddagger \S$ & $47.9 \pm 3.0 \dagger \ddagger \S$ & $40.1 \pm 4.1^{*}$ \\
\hline LVEDV & $0.542 \pm 0.053$ & $0.519 \pm 0.050$ & $0.492 \pm 0.038$ & $0.438 \pm 0.019$ & $0.429 \pm 0.023$ \\
\hline LVESV & $0.328 \pm 0.047$ & $0.312 \pm 0.030^{*}$ & $0.251 \pm 0.026$ & $0.230 \pm 0.019$ & $0.246 \pm 0.019 *$ \\
\hline HR (echo) & $358 \pm 5$ & $368 \pm 8$ & $355 \pm 5$ & $355 \pm 11$ & $357 \pm 8$ \\
\hline BW & $234 \pm 3^{*}$ & $230 \pm 4^{*}$ & $235 \pm 4^{*}$ & $234 \pm 3^{*}$ & $236 \pm 2^{*}$ \\
\hline $\mathrm{dP} / \mathrm{dt}_{\max }$ & $4027 \pm 199$ & $3908 \pm 248$ & $4960 \pm 266 \dagger \ddagger$ & $4748 \pm 304 \dagger \ddagger$ & $4401 \pm 229$ \\
\hline $\mathrm{dP} / \mathrm{dt}_{\min }$ & $-3964 \pm 205$ & $-3661 \pm 258$ & $-4751 \pm 319 \dagger \ddagger$ & $-4705 \pm 354 \ddagger$ & $-4227 \pm 286$ \\
\hline LVESP & $113 \pm 3$ & $110 \pm 5$ & $123 \pm 5$ & $126 \pm 7$ & $115 \pm 6$ \\
\hline LVEDP & $13.3 \pm 4.0$ & $13.9 \pm 4.0$ & $13.4 \pm 2.2$ & $10.2 \pm 1.5$ & $7.9 \pm 1.2$ \\
\hline HR (cath) & $335 \pm 10$ & $328 \pm 9$ & $340 \pm 7$ & $334 \pm 8$ & $317 \pm 9$ \\
\hline
\end{tabular}

No MI, Normal rats without myocardial infarction; Sham, sham operation; $P U$, implantation of nonseeded polyurethane patch; $P U-M y o B$, myoblast-seeded polyurethane patch; Medium, contrast medium only; $E F$, ejection fraction $(\%) ; L V E D V$, left ventricular end-diastolic volume $(\mathrm{mL}) ; \angle V E S V$, left ventricular end-systolic volume $(\mathrm{mL}) ; H R$ (echo), heart rate measured echocardiographically $(1 / \mathrm{min}) ; B W$, body weight $(\mathrm{g}) ; d P / d t_{\text {max }}$ left ventricular contractility $(\mathrm{mm}$ $\mathrm{Hg} / \mathrm{s}) ; d P / d t_{\text {min, }}$ left ventricular relaxation $(\mathrm{mm} \mathrm{Hg} / \mathrm{s}) ; L V E S P$, left ventricular end-systolic pressure $(\mathrm{mm} \mathrm{Hg}) ; L V E D P$, left ventricular end-diastolic pressure $(\mathrm{mm} \mathrm{Hg}) ; H R($ cath), heart rate measured with pressure-tip catheter $(1 / \mathrm{min})$. Values are mean $\pm \mathrm{SE} . * P<.05$ versus pre; $\dagger P<.05$ versus Sham; $\ddagger P<$ .05 versus $\mathrm{PU} ; \S P<.05$ versus Medium. 


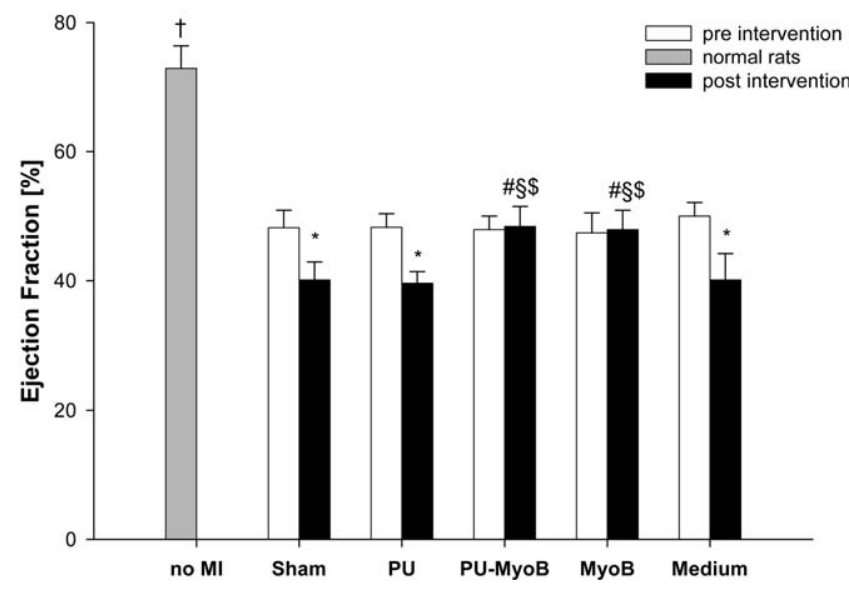

Figure 1. Left ventricular ejection fraction (LVEF [\%]). Gray bar, No MI, healthy animals without myocardial infarction; Sham, animals with infarction but without intervention; $P U$, nonseeded polyurethane scaffold implantation; $P U-M y o B$, scaffolds seeded with myoblasts; $M y o B$ Inj, direct intramyocardial injection; $M e$ dium, medium injection. ${ }^{*} P<.05$ versus preintervention in the same group; $\# \boldsymbol{P}<.05$ versus Sham; $\$ P<.05$ versus $\mathrm{PU} ; \boldsymbol{P}<.05$ versus Medium; $+P<.05$ versus all groups preinterventional and postinterventional.

In all animals the MI was confirmed by hematoxylineosin staining. Additionally, immunostaining with anti- $\alpha$ actinin and anti-slow-muscle myosin demonstrated viable myocardium surrounding the infarction zone (Figure $5, b$ and $c$ ).

In the animals injected with skeletal myoblasts, a differentiation of the engrafted cells to myotubes was evidenced by a positive staining for anti-skeletal myosin (fast), which was distributed regularly within the infarction zone (Figure $5, d)$.

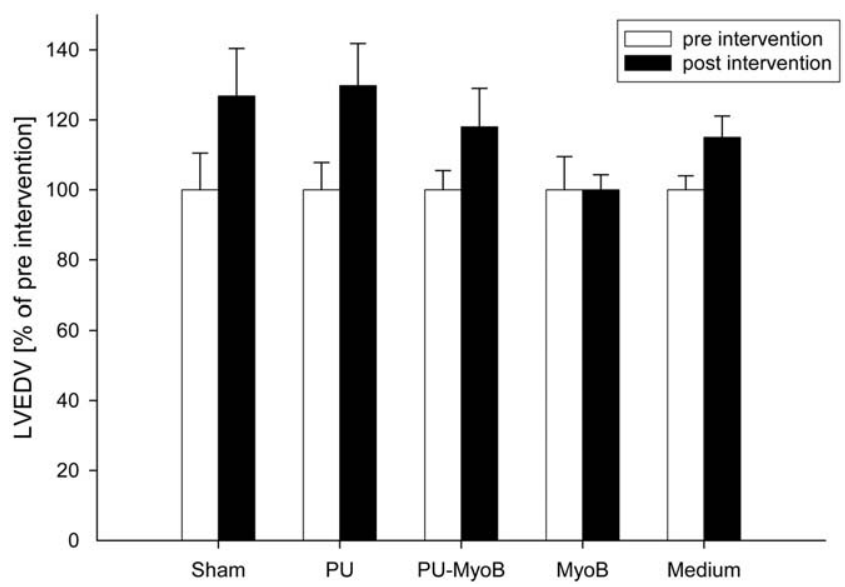

Figure 2. Left ventricular end-diastolic volume (LVEDV) in percent of the preinterventional values. For abbreviations, see Figure 1.

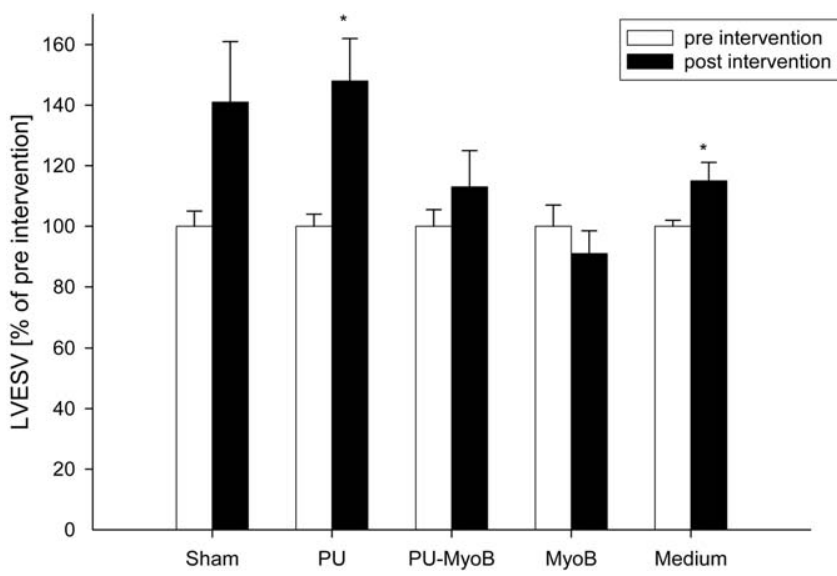

Figure 3. Left ventricular end-systolic volume (LVESV) in percent of the pre-interventional values. For abbreviations, see Figure 1.

Macroscopically, the scaffolds in the PU-MyoB and PU groups were not degraded and were strongly sticking to the infarcted surface. Neo-vessels could be noticed within the adhesion tissue surrounding the scaffold (Figure 5, a). Histologic analysis of hematoxylin-eosin-stained sections revealed a dense cellular colonization including vascularization of the scaffolds in both groups. The scaffold material itself showed no histologic signs of degradation. No inflammatory reaction of the neighboring host tissue nor encapsulation of the graft was detected .The mononuclear cells were randomly oriented. We detected neither myotubes (negative staining for anti-(fast)-skeletal myosin) nor ingrowths of myocardium (negative staining for anti- $\alpha$-actinin and antislow-muscle myosin) inside the grafts (Figure $5, e$ ). In the

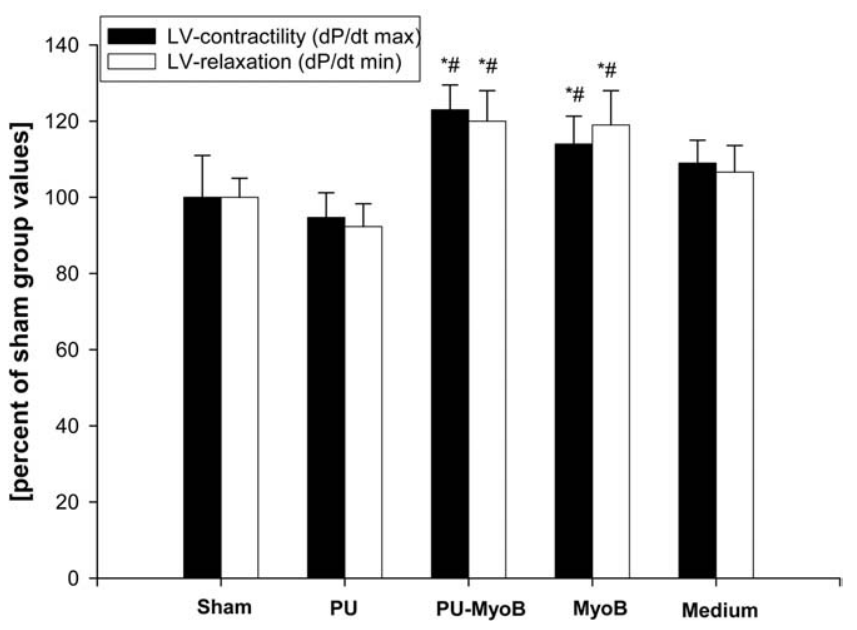

Figure 4. Left ventricular contractility $\left(\mathrm{dP} / \mathrm{dt}_{\max }\right)$ and relaxation $\left(\mathrm{dP}_{\mathrm{d}} \mathrm{dt}_{\min }\right)$. For abbreviations, see Figure 1. ${ }^{*} \boldsymbol{P}<.05$ versus Sham, $\# P<.05$ versus $P U$. 

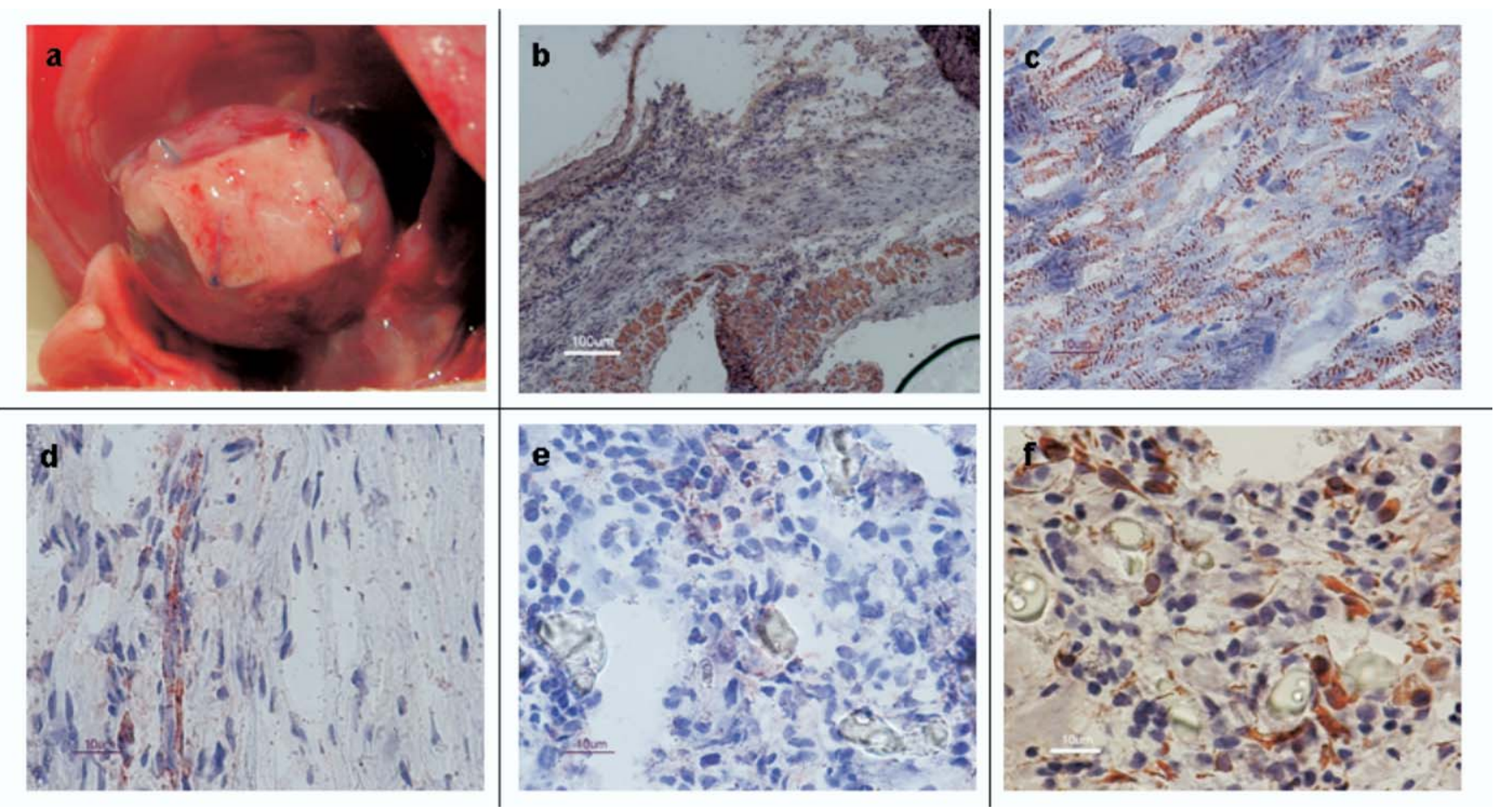

Figure 5. Macroscopy and immunohistology 4 weeks after intervention. a, Macroscopic view of the implanted scaffold (PU-MyoB group). Note the visible vessels running toward the patch from the posterior wall. b, Infarcted myocardium (Sham group) surrounded by viable endocardial and epicardial myocytes visualized by anti-slowmyosin immunostaining $(100 \times)$. c, Viable myocardium bordering the infarction zone (Sham group) evidenced by anti-actinin immunostaining $(600 \times)$. Note the striation of the living cardiomyocyte filaments. d, Islet of engrafted myotubes in the infarction zone (MyoB group) stained by anti-fast-myosin antibody $(600 \times)$. e, Immunostaining using anti-skeletal myosin $(600 \times)$. Inside the PU scaffold (PU-MyoB group) the cells are randomly oriented. No skeletal myosin positivity can be detected suggesting missing differentiation to myotubes of the grafted cells. $f$, Immunostaining using anti-desmin antibodies to visualize the myogenic progenitor cells (PU-MyoB) spreading undifferentiated throughout the scaffold in great numbers $(600 \times)$.

animals implanted with myoblast-based scaffolds, only desmin-positive cells were detected in great numbers throughout the scaffolds (Figure 5, f). As expected, no desmin-positive cells were detected in the control nonseeded scaffolds. Interestingly, 4 weeks after in vivo implantation of the PU MyoB, the desmin-positively stained cells remained isolated from each other and did not show any intracellular striation, suggesting they had retained an undifferentiated pattern. In addition, we found no penetration of these scaffold-bound cells into the neighboring myocardium or infarction zone.

\section{Discussion}

This study demonstrates the potential value of approaching the therapy of ischemic heart failure by engrafting a bioengineered cell-seeded scaffold. The most salient findings of this study are as follows: (1) Myoblast-seeded PU scaffolds prevent further deterioration of left ventricular func- tion after MI compared with placebo-injected controls, but (2) they do not yield a better preservation of left ventricular function than myoblasts directly injected into the scarred myocardium; (3) even though the number of animals tested was small, the implantation of a myoblast-seeded scaffold after an MI seemed to reduce the mortality, inasmuch as none of these animals died during the 4 weeks' observation period as compared with a mortality rate of $20 \%$ observed in the groups that did not receive myoblasts.

The initial results of phase I clinical trials of skeletal myoblast transplantation have provided encouraging hints, ${ }^{4-6}$ although clear-cut evidence for efficacy is still lacking and will only be provided by randomized studies, one of which is already ongoing. However, regardless of the putative benefits of the procedure, it is likely that its efficacy still remains limited by the high rate of early posttransplantation cell death ${ }^{7-9}$ and the inability of the subsequent proliferation of the surviving cells to reverse the initial graft 
attrition. ${ }^{15}$ Although cell loss is a multifactorial process, physical strain related to the multiple injections is usually identified as a contributory factor. This has provided a rationale for testing myoblast-seeded scaffolds that could represent a less invasive and more expeditious means of delivering the candidate cells to the target scar. A comparable approach has been tried by some groups using fibroblasts or bone marrow cells bound to different scaffolds, mainly achieving a benefit through neovascularization. ${ }^{16-18}$ Being aware of the properties of myoblasts to restore myocardial function, we transferred this concept to myoblastbased scaffolds.

However, very interestingly, we observed that despite the high number of cells that could be brought using this technique ( $\sim 80 \%$ of initial cells applied: $3.9 \pm 0.5 \times 10^{6}$ viable cells), we were not able to increase the functional benefit as compared with the direct injection approach (10\%-20\% of the initial cells applied survive the injection process). This raises several questions, one being that the number of transplanted cells, although probably important, ${ }^{19}$ may not be as critical as initially thought, at least regarding the contractile function. Consequently, other mechanisms are certainly involved.

The histologic observations that myoblasts bound to scaffolds retained an undifferentiated phenotype and, more important, did not migrate toward the diseased area probably account for the inability of these "entrapped" cells to actively participate in contraction and/or relaxation and their resulting failure to elicit a superior functional outcome. At the same time, these data provide some mechanistic insights in that they suggest that the benefits seen in the PU-MyoB group compared with sham or control animals were likely related to some paracrine signaling independent of the intrinsic contractile properties of myoblasts. ${ }^{9,20-22}$ Microarray gene studies and subsequent proteomic analyses, currently underway in our laboratory, are now trying to identify the myoblast-derived factors that may act on angiogenesis, extracellular matrix remodeling, apoptosis, or resident cardiac cell proliferation and account for the benefits of these cells on heart function, even though they remained epicardially located.

Furthermore, the improved recovery patterns seen in the myoblast-seeded PU group (PU-MyoB) compared with the myoblast-free control PU counterparts also suggest that biologic patches may be more effective than exclusively synthetic passive constraint devices for limiting postinfarction left ventricular remodeling and the ensuing deterioration of heart function. The observation that directly injected myoblasts were more effective than myoblasts seeded on PU matrices in preserving postinfarction left ventricular geometry could be related to the physical properties of our PU scaffold or to the fact that it simply overlaid the scar area and was therefore not able to exert the girdling effect that may result from a more complete wrapping of the entire left ventricle.

Limitations of this study include the relatively brief period of follow-up that precludes assessing a potential later myoblast differentiation as well as the lack of identification of the growth factors/cytokines that may have mediated the functional effects of the seeded myoblasts. Furthermore, the myoblast purity we obtained (about 33\%), even if in accordance with others (Leobon and associates ${ }^{11} 34 \%$, Azarnoush and associates ${ }^{23} 38 \%$ ) remains relatively low. Consequently, the real contribution of the myoblasts to the functional effect can obviously be questioned. Indeed, the effect of a pure fibroblast preparation was not tested in our study. Finally, it also remains to assess whether the intramyocardial injections of chemoattractants like stromaderived factor $1^{24}$ could enhance migration of the superficially deposited myoblasts, promote their intramyocardial engraftment, and foster cardiac tissue repair.

In conclusion, this study raises a cautionary note about the putative superiority of scaffold-seeded myoblasts above the direct injection technique and provides indirect mechanistic evidence for the paracrinally mediated effects of these cells on the postinfarction improvement in functional outcomes.

Artelon-PU scaffold material was kindly supplied by R\&D Department of Artimplant AB (Västra Frölunda, Sweden; person of contact: Ulf Akerblom) with scientific support from Elisabeth Liljensten.

\section{References}

1. Murry CE, Wiseman RW, Schwartz SM, Hauschka SD. Skeletal myoblast transplantation for repair of myocardial necrosis. J Clin Invest. 1996;98:2512-23.

2. Pouzet B, Ghostine S, Vilquin JT, Garcin I, Scorsin M, Hagege AA, et al. Is skeletal myoblast transplantation clinically relevant in the era of angiotensin-converting enzyme inhibitors? Circulation. 2001; 104(suppl I):I223-8.

3. Taylor DA, Atkins BZ, Hungspreugs P, Jones TR, Reedy MC, Hutcheson KA, et al. Regenerating functional myocardium: improved performance after skeletal myoblast transplantation. Nat Med. 1998; 4:929-33.

4. Smits PC, van Geuns RJ, Poldermans D, Bountioukos M, Onderwater $\mathrm{EE}$, Lee $\mathrm{CH}$, et al. Catheter-based intramyocardial injection of autol ogous skeletal myoblasts as a primary treatment of ischemic heart failure: clinical experience with six-month follow-up. J Am Coll Cardiol. 2003;42:2063-9.

5. Siminiak T, Kalawski R, Fiszer D, Jerzykowska O, Rzezniczak J, Rozwadowska N, et al. Autologous skeletal myoblast transplantation for the treatment of postinfarction myocardial injury: phase I clinical study with 12 months of follow-up. Am Heart J. 2004;148:531-7.

6. Menasche P, Hagege AA, Scorsin M, Pouzet B, Desnos M, Duboc D, et al. Myoblast transplantation for heart failure. Lancet. 2001;357:27980 .

7. Fan Y, Maley M, Beilharz M, Grounds M. Rapid death of injected myoblasts in myoblast transfer therapy. Muscle Nerve. 1996;19:85360.

8. Zhang M, Methot D, Poppa V, Fujio Y, Walsh K, Murry CE. Cardiomyocyte grafting for cardiac repair: graft cell death and anti-death strategies. J Mol Cell Cardiol. 2001;33:907-21. 
9. Suzuki K, Murtuza B, Beauchamp JR, Smolenski RT, Varela-Carver A, Fukushima S, et al. Dynamics and mediators of acute graft attrition after myoblast transplantation to the heart. Faseb J. 2004;18:1153-5.

10. Siepe M, Giraud M, Tevaearai H, Nydegger U, Menasché P, Carrel T. Biodegradable polyurethane scaffold seeded with myoblasts for myocardial repair. ASAIO J. 2004;50(abstr.):160.

11. Leobon B, Garcin I, Menasche P, Vilquin JT, Audinat E, Charpak S. Myoblasts transplanted into rat infarcted myocardium are functionally isolated from their host. Proc Natl Acad Sci U S A. 2003;100:7808-11.

12. Pouzet B, Vilquin JT, Hagege AA, Scorsin M, Messas E, Fiszman M, et al. Intramyocardial transplantation of autologous myoblasts: can tissue processing be optimized? Circulation. 2000;102(suppl 3):III210-5.

13. Gisselfalt K, Edberg B, Flodin P. Synthesis and properties of degradable poly(urethane urea)s to be used for ligament reconstructions. Biomacromolecules. 2002;3:951-8.

14. Siepe M, Rüegg M, Giraud M, Python J, Carrel T, Tevaearai H. Effect of acute body positional changes on the hemodynamics of rats with and without myocardial infarction. Exp Physiol. 2005;90:627-34.

15. Maurel A, Azarnoush K, Sabbah L, Vignier N, Le Lorc'h M, Mandet $\mathrm{C}$, et al. Can cold or heat shock improve skeletal myoblast engraftment in infarcted myocardium? Transplantation. 2005;80:660-5.

16. Kellar RS, Landeen LK, Shepherd BR, Naughton GK, Ratcliffe A, Williams SK. Scaffold-based three-dimensional human fibroblast culture provides a structural matrix that supports angiogenesis in infarcted heart tissue. Circulation. 2001;104:2063-8.

17. Fukuhara S, Tomita S, Nakatani T, Fujisato T, Ohtsu Y, Ishida M, et al. Bone marrow cell-seeded biodegradable polymeric scaffold enhances angiogenesis and improves function of the infarcted heart. Circ J. 2005;69:850-7.
18. Barandon L, Couffinhal T, Dufourcq P, Alzieu P, Daret D, Deville C, et al. Repair of myocardial infarction by epicardial deposition of bone-marrow-cell-coated muscle patch in a murine model. Ann Thorac Surg. 2004;78:1409-17.

19. Pouzet B, Vilquin JT, Hagege AA, Scorsin M, Messas E, Fiszman M, et al. Factors affecting functional outcome after autologous skeletal myoblast transplantation. Ann Thorac Surg. 2001;71:844-50; discussion 50-1.

20. Murtuza B, Suzuki K, Bou-Gharios G, Beauchamp JR, Smolenski RT, Partridge TA, et al. Transplantation of skeletal myoblasts secreting an IL-1 inhibitor modulates adverse remodeling in infarcted murine myocardium. Proc Natl Acad Sci U S A. 2004;101:4216-21.

21. Suzuki K, Murtuza B, Beauchamp JR, Brand NJ, Barton PJ, VarelaCarver A, et al. Role of interleukin-1beta in acute inflammation and graft death after cell transplantation to the heart. Circulation. 2004; 110(suppl 1):II219-24.

22. Kamihata H, Matsubara H, Nishiue T, Fujiyama S, Tsutsumi Y, Ozono $\mathrm{R}$, et al. Implantation of bone marrow mononuclear cells into ischemic myocardium enhances collateral perfusion and regional function via side supply of angioblasts, angiogenic ligands, and cytokines. Circulation. 2001;104:1046-52.

23. Azarnoush K, Maurel A, Sebbah L, Carrion C, Bissery A, Mandet C, et al. Enhancement of the functional benefits of skeletal myoblast transplantation by means of coadministration of hypoxia-inducible factor 1 $\alpha . J$ Thorac Cardiovasc Surg. 2005;130:173-9.

24. Askari AT, Unzek S, Popovic ZB, Goldman CK, Forudi F, Kiedrowski $\mathrm{M}$, et al. Effect of stromal-cell-derived factor 1 on stem-cell homing and tissue regeneration in ischaemic cardiomyopathy. Lancet. 2003; 362:697-703. 